\title{
Kikuchi-Fujimoto disease (KFD): A Case Report
}

\author{
Islam A. M. Abu-Nayla ${ }^{1}$, Abdulameer M. Abu Nailah ${ }^{2}$ \\ and Umniyah A. M. Abu-Nayla ${ }^{3}$
}

\begin{abstract}
Kikuchi-Fujimoto disease (KFD) is a rare entity characterized by subacute necrotizing lymphadenopathy. Despite many studies in the literature, the cause of KFD remains uncertain. Young adults of Asian ancestry are most affected, but it has been reported worldwide. Clinically, KFD is characterized by lymphadenitis of one or more lymph nodes, predominantly in the posterior cervical region, fever, and leukopenia in up to $50 \%$ of cases. In this case report, we are presenting a case of Kikuchi-Fujimoto disease in a young Filipino lady.
\end{abstract}

Keywords: Kikuchi-Fujimoto disease, KFD, Kikuchi disease, Acute necrotizing lymphadenopathy, Lymphadenitis.

\footnotetext{
${ }^{1}$ Urology Registrar, Southmead Hospital, Bristol, UK.

2 Specialist Rheumatologist MBChB, F.I.C.M, F.I.C.M.S Rheumatology and medicine rehabilitation, MRCP (Rheumatology) Canadian Specialist Hospital, Dubai, UAE.

${ }^{3}$ Medical Intern, Dubai Health Authority (DHA), UAE.
} 


\section{Introduction}

Kikuchi-Fujimoto Disease is an enigmatic, benign and self-limited syndrome characterized by regional lymphadenopathy with tenderness, predominantly in the cervical region, usually accompanied by mild fever and night sweats. Initially described in Japan, KFD was first reported in 1972 almost simultaneously by (Kikuchi M, 1972) and by (Fujimoto et al, 1972). Kikuchi-Fujimoto disease is typically self-limited within one to four months, a low but possible recurrence rate of 3 to $4 \%$ has been reported by (Dorfman, 1987). The clinical, histopathological and immunohistochemical features appear to point to a viral etiology, a hypothesis that still has not been proven. We Reported this rare case in the Rheumatology Department.

\section{Case Report}

1. A 24 year old Pilipino lady admitted in February 2019 with right -sided cervical lymph node enlargement, associated with fever. The patient reported small bulges along the right side of her neck for one month. There is fever (38.3-38.9), fatigue and diffuse joint pain. She was treated by her general practitioner with 2 courses of orally administered antibiotic to which she did not respond. Furthermore, the patient complained of backache, mild upper abdominal pain, headache, and severe anorexia and lost $3 \mathrm{~kg}$ over the period of 1 month. The patient denied any history of chronic cough, joint swelling, sore throat or skin rash. She denied sick contacts, recent travel or exposure to pets. The patient has family history of non-Hodgkin lymphoma (her sister), no other significant family history of note.

a) On examination, there were multiple palpable tender right-sided cervical lymph nodes, around $2 \times 1.5 \mathrm{~cm}$, without evidence of any erythema, oedema, or warmth of the overlying skin. There was mild tenderness of the joint line of the inter-phalangeal joints bilaterally, not associated with any swelling. Rest of the systemic examination was unremarkable.

2. Laboratory findings showed mild lymphopenia (White Blood Cell count $6.2 \times$ 109/L with $72 \%$ neutrophils, 19\% lymphocytes, $7.3 \%$ monocytes, $0.6 \%$ Eosinophils, and $0.1 \%$ basophils). ESR was only mildly elevated at $21 \mathrm{~mm} / \mathrm{hr}$, $\mathrm{C}$ reactive protein $7.5 \mathrm{mg} / \mathrm{l}$. Urine and blood cultures were negative. The patient had a negative infectious disease workup that included monospot test, EBV IgM, tuberculosis (interferon-gamma release assays), HIV (PCR and viral load) and Hepatitis B \&C. Anti-nuclear Antibodies were negative.

3. Ultrasound of the neck was initially done revealing sub-centimeter, reactive normal lymph nodes seen in bilateral level II and level III. There were few lymph nodes seen in right side of neck which were round, hypoechoic with 
thin hilum and minimal vascularity measuring $1.5 \times 0.6 \mathrm{~cm}$ and $0.9 \times 0.5 \mathrm{~cm}$ at level II, $0.7 \times 0.4 \mathrm{~cm}, 0.9 \times 0.5 \mathrm{~cm}$ at level III and $1.2 \times 0.6 \mathrm{~cm}$ at level IV. Thyroid gland was normal. Chest $\mathrm{X}$ ray and abdominal ultrasound revealed normal study.

4. An excisional lymph node biopsy from the anterior cervical chain was performed and on histopathological analysis Sections reveal multiple fragments of a lymph node with foci of necrosis and abundant aggregates of histiocytes. No typical Reed-Sternberg cells are seen. No anaplastic features are identified. Therefore, the histopathological analysis and the immunohistochemistry study results are in keeping with the diagnosis of Kikuchi disease

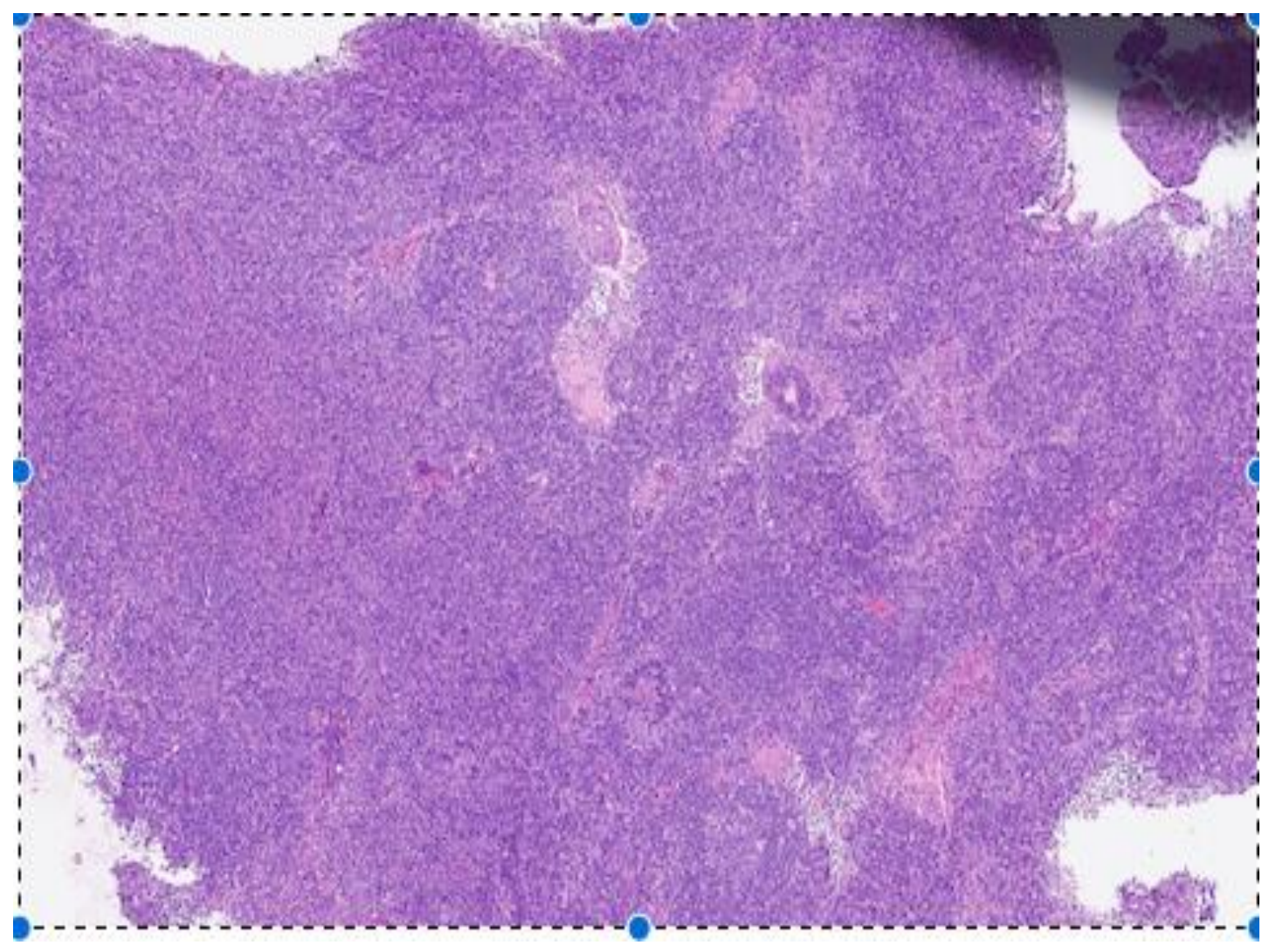

Figure 1: histopathological analysis reveals multiple fragments of a lymph node with foci of necrosis and abundant aggregates of histiocytes. 


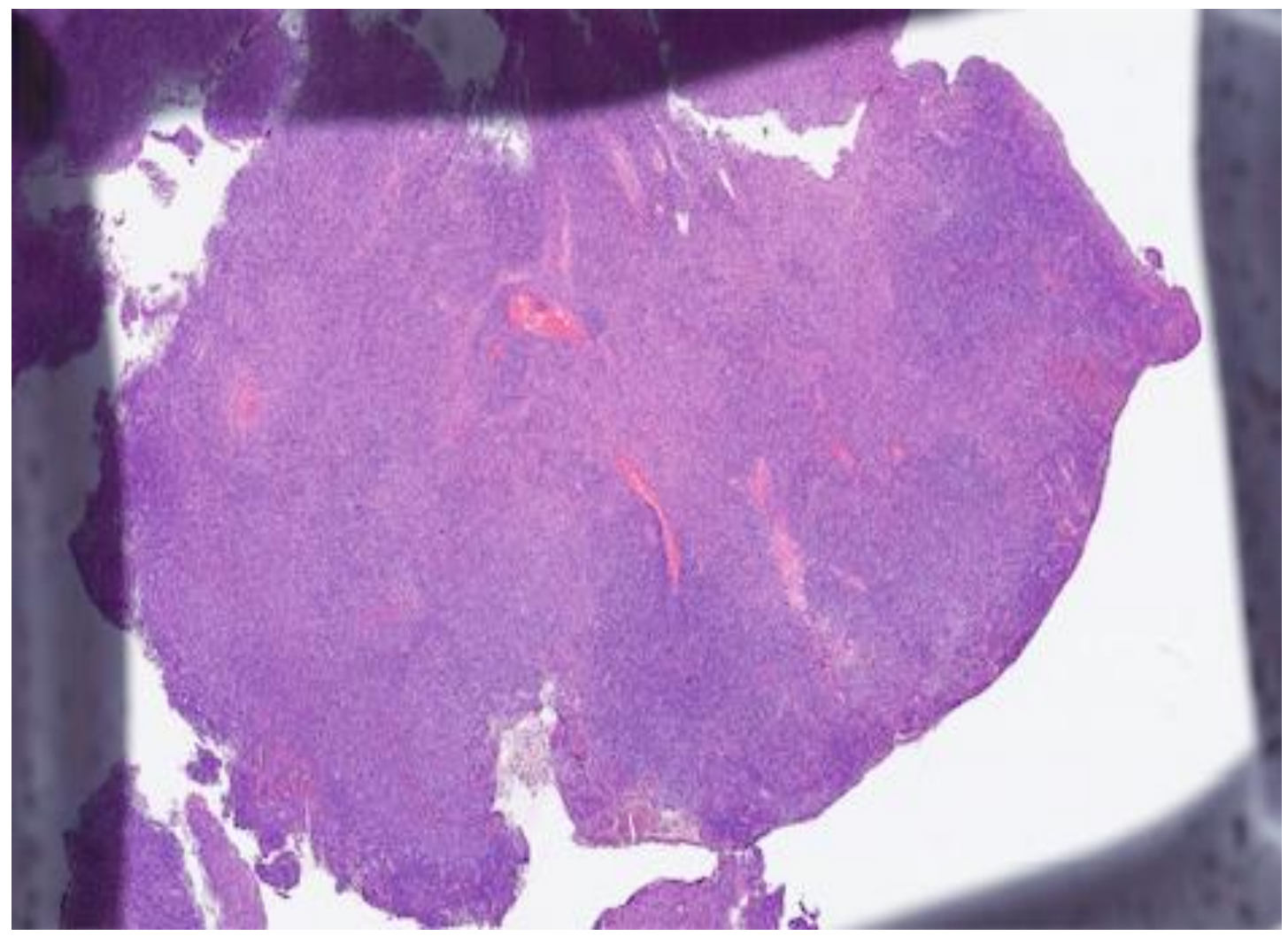

Figure 2: Another view of histopathological analysis that also reveal multiple fragments of a lymph node with foci of necrosis and abundant aggregates of histiocytes.

5. In addition, immunohistochemistry study was revealed: CD3 and CD20: polyclonal phenotype, CD68: positive in histiocytic infiltrate, CD30: positive in few scattered cells, CD15: positive in few scattered cell, Ki 67: reactive pattern

\section{Conclusions}

Kikuchi-Fujimoto disease is an extremely rare disease known to have a worldwide distribution with a higher prevalence among Japanese and other Asiatic individuals as mentioned by (Bosch et al, 2004). Affected patients are most often young adults under the age of 30 years; the disease is seldom reported in children. There is much speculation about the etiology of KFD. A viral or autoimmune cause has been suggested. The role of Epstein-Barr virus, as well as other viruses (HHV6, HHV8, parvovirus B19) in the pathogenesis of KFD remains controversial and not convincingly demonstrated. A viral infection is, nonetheless, possible by virtue of clinical manifestations, as described by (Unger et al, 1987). that include upper 
respiratory prodrome, atypical lymphocytosis and lack of response to antibiotic therapy, and certain histopathologic features (i.e., T-cells as revealed by immunological marker studies). Although the mechanism of cell death involved in KFD has not been extensively studied, (Ohshima et al, 1998). have shown that apoptotic cell death may play a role in the pathogenesis of the disease. According to these authors, proliferating CD8 positive T-cells may act as "killers" and "victims" in the apoptotic process via Fas -and perforine- pathways.

30 to $50 \%$ of patients with KFD may have fever, usually of low-grade, associated with upper respiratory symptoms. Less frequent symptoms include weight loss, nausea, vomiting, sore throat and night sweats as described by (Yen et al, 1997); (Bosch et al, 2004). Leukopenia can be found in up to 50\% of the cases. KFD has also been reported as a cause of pro longed fever of unknown origin stated by (Kapadia et al, 1989).

The onset of KFD is acute or subacute, evolving over a period of two to three weeks. Cervical lymphadenopathy is almost always present consisting of tender lymph nodes that involve mainly the posterior cervical triangle. Lymph node size has been found to range from 0.5 to $4 \mathrm{~cm}$, but it may reach 5 to $6 \mathrm{~cm}$ and rarely larger than 6 $\mathrm{cm}$. Generalized lymphadenopathy can occur but is very rare verified by (Kuo, 1995); (Lin et al, 2003).

Kikuchi-Fujimoto disease is generally diagnosed based on an excisional biopsy of affected lymph nodes. No specific diagnostic laboratory tests are available. The results of a wide range of laboratory studies are usually normal. Nevertheless, some patients have anemia, slight elevation of the erythrocyte sedimentation rate and even leukopenia. Of note, one third of patients present atypical peripheral blood lymphocytes, (Lin et al, 2003). Characteristic histopathologic findings of KFD include irregular paracortical areas of coagulative necrosis with abundant karyorrhectic debris, which can distort the nodal architecture, and large number of different types of histiocytes at the margin of the necrotic areas. The karyorrhectic foci are formed by different cellular types, predominantly histiocytes and plasmacytoid monocytes but also immunoblasts and small and large lymphocytes. Neutrophils are characteristically absent and plasma cells are either absent or scarce. Importantly, atypia in the reactive immunoblastic component is not uncommon and can be mistaken for lymphoma, (Tsang et al, 1994). The immunophenotype of KFD typically consists of a predominance of T-cells, with very few B-cells. There is an abundance of CD8+ T-cells over CD4+. The histiocytes express histiocyteassociated antigens such as lysozyme, myeloperoxidase (MPO) and CD68.

Finally, striking plasmacytoid monocytes are also positive for CD68 but not for MPO, (Bosch et al, 2004).

Kikuchi-Fujimoto disease is typically self-limited within one to four months. A low but possible recurrence rate of 3 to $4 \%$ has been reported, (Pileri et al, 2005). In some few patients, SLE may occur some years later. No risk to other family members is felt to be associated with KFD. Symptomatic measures aimed to relief the distressing local and systemic complains should be employed. Analgesicsantipyretics and nonsteroidal anti-inflammatory drugs may be used to alleviate 
lymph node tenderness and fever. The use of corticosteroids has been recommended in severe extranodal or generalized KFD but is of uncertain efficacy. Surgical consultation may be indicated for a diagnostic excisional lymph node biopsy. Patients with KFD require a systematic survey and regular follow-up for several years to rule out the development of SLE. The cervical lymphadenopathy runs a benign course and appears to resolve spontaneously 1 to 6 months after definite diagnosis.

ACKNOWLEDGEMENTS: We thank the patient and his family members for providing consent and for his participation in the case.

\section{References}

[1] Kikuchi, M. (1972). Lymphadenitis showing focal reticulum cell hyperplasia with nuclear debris and phagocytes: a clinicopathological study. Acta Hematol Jpn (1972), 35:379-380.

[2] Fujimoto, Y., Kozima, Y., Yamaguchi, K. (1972).

Cervical subacute necrotizing lymphadenitis: a new clinicopathologic entity. Naika (1972),20:920-927.

[3] Dorfman, R.F. (1987). Histiocytic necrotizing lymphadenitis of Kikuchi and Fujimoto. Arch Pathol Lab Med (1987), 111:1026-1029.

[4] Bosch, X., Guilabert, A., Miquel, R., Campo, E. (2004).

Enigmatic Kikuchi-Fuji- moto disease: a comprehensive review. Am J Clin Pathol (2004),122:141-152.

[5] Unger, P.D., Rappaport, K.M., Strauchen, J.A. (1987). Necrotizing lymphadenitis (Kikuchi's disease). Report of four cases of an unusual pseudolymphomatous lesion and immunologic marker studies. Arch Pathol LabMed (1987), 111:1031-1034

[6] Ohshima, K., Shimazaki, K., Kume, T., Suzumiya, J., Kanda, M., Kikuchi, M. (1998). Perforin and Fas pathways of cytotoxic T-cells in histiocytic necrotizing lymphadenitis. Histopathology (1998), 33:471-478.

[7] Yen, A., Fearneyhough, P., Raimer, S.S., Hudnall, S.D. (1997).

EBV-associated Kikuchi's histiocytic necrotizing lymphadenitis with cutaneous manifestations. J Am Acad Dermatol (1997), 36:342-346.

[8] Bosch, X., Guilabert, A. (2004). Kikuchi-Fujimoto disease. Med Clin (Barc) (2004), 123:471-476.

[9] Kapadia, Y., Robinson, B.A., Angus, H.B. (1989). Kikuchi's disease presenting as fever of unknown origin. Lancet (1989), 2:1519-1520.

[10] Kuo, T. (1995). Kikuchi's disease (histiocytic necrotizing lymphadenitis). A clinicopathologic study of 79 cases with an analysis of histologic subtypes, immunohistology, and DNA ploidy. Am J Surg Pathol (1995), 19:798-809.

[11] Lin, H.C., Su, C.Y., Huang, C.C., Hwang, C.F., Chien, C.Y. (2003). Kikuchi's disease. A review and analysis of 61 cases. Otoralyngol Head Neck Surg (2003), 128:650-653. 
[12] Tsang, W.Y.W., Chan, J.K.C., Ng, C.S. (1994). Kikuchi's lymphadenitis. A morphologic analysis of 75 cases with special reference to unusual features. Am J Surg Pathol (1994), 18:219-231.

[13] Pileri, A.S., Pileri, A., Yasukawa, K., Kuo, T.S., Sullivan, K. 92005). The Karma of Kiluchi's disease. Clin Immunol (2005), 114:27-29. 